\title{
PUBLIC PARTICIPATION IN GIS VIA MOBILE APPLICATIONS FOR CRISIS MANAGEMENT PROCESS: A CASE STUDY OF AN EARTHQUAKE, TEHRAN, IRAN
}

\author{
S. Farhadpour ${ }^{1}$, F. Hosseinali ${ }^{*}, 1$ \\ ${ }^{1}$ Dept. of Surveying Engineering, Faculty of Civil Engineering, Shahid Rajaee Teacher Training University, Lavizan, Tehran, Iran - \\ sarah.fr.72@gmail.com, f.hosseinali@sru.ac.ir
}

KEY WORDS: Earthquake, Crisis Management, Vulnerability Map, Fuzzy Inference, Internet Connection, Mobile GIS, VGI

\begin{abstract}
:
The effects of earthquakes on societies are greater than what most people think. These effects range from structural damages to economic impacts and fatalities. An earthquake only lasts for a few seconds and the aftershocks may continue for days, but the damage does continue for years. The lack of integrated and automated tools for estimating resources needed in the aftermath of an earthquake during the disaster response phase motivate the design and implementation of a comprehensive, interactive, and userfriendly model. The objective of this research is to use geospatial analyses for examining vulnerable areas and evaluating the hazard of building damage by earthquake. Hence, the effective parameters of damage are identified and by using the fuzzy inference, the degree of damage in the study area is determined. Subsequently, with the provision of internet service in a troubled region, it is possible to collect voluntary information from people in the region using their mobile smartphones. For this purpose, an app in the Android Studio environment has been developed for convenience and sending the exact location of the affected sites. Moreover, the result of this research identified areas with a high priority for relief and optimal use of time, especially in the early hours of the crisis.
\end{abstract}

\section{INTRODUCTION}

Iran is one of the countries with high intensity of earthquake. The main reason for this matter is the location of Iran between three main plates namely: Arabian plate at the Southwest, Indian plate at the East and Southeast and Siberian plate at the Northeast (Zare and Kamran Zad, 2015). Unknown risk, inappropriate disaster management, high exposure of element at risk, and vulnerable buildings are four major factors that can lead to increased casualties and loss of property in earthquake event (Hassanzadeh et al., 2013). For example the Bam city earthquake in Iran (2003) resulted in the death of more than 30,000 people and the destruction of infrastructures and many buildings, because the seismic risk at Bam city was underestimated (Nadim et al., 2004), and there was no plan for disaster management (Alavi Razavi, 2008).

With the fast development of mobile Web and computing technologies, as well as increasing availability of mobile devices, mobile information technologies have revolutionary influence the human society (Gao and Mai, 2018). In the domain of Geographic Information Systems (GIS), advanced mobile information technologies have lowered the traditional enterprise GIS fence and enabled a variety of novel applications which can help improve positioning and tracking accuracy, efficient field data collection, ground truth validation, location intelligence and decision support, and so on (Abdalla, 2016; Lemmens, 2011). The revolution brought by this new trend has been traditionally associated with the term Volunteered Geographic Information (VGI), that Goodchild coined (2007a) and explained (2007b) by comparing humans to "intelligent, mobile sensors" able to acquire precious geospatial information of unparalleled depth in both a spatial dimension and a temporal dimension. Another successful term that is widely used in GIS literature is geocrowdsourcing (Goetz and Zipf, 2013) or simply crowdsourcing (Geng et al., 2016; Hudson-Smith et al., 2008), involving the collection of geospatial information performed by an undefined network of people. However, even though VGI and crowdsourcing have slightly different underlying meanings, they are usually treated as synonyms or even combined (Peterson, 2013).

This paper is focused on creating a vulnerability map using fuzzy inference method for estimating the degree of destruction. In the fuzzy inference method before the earthquake, the amount of seismic physical vulnerability of each building can be estimated relatively well. Then, establishment of an internet connection and data collection from the crisis region is considered. A mobile application is developed which enables the amateurs to easily ask for help using their smart cell phones. Smart phones have played a significant role in shaping the technological innovation. Being directly connected to the internet and equipped with not only a GPS receiver but also a huge number of other sensors, they allow users to easily acquire and share geospatial contents and thus represent the foundation of many Volunteered Geographic Information (VGI) and participatory sensing activities.

\section{PROPOSED METHOD}

In this study, fuzzy inference method was used to classify the degree of damage of residential buildings in the study area. Spatial and descriptive data in fuzzy method are classified in different classes in terms of degree of damage (or vulnerability against the earthquake). Then, by applying the fuzzy rules, the vulnerability map is obtained and the amount of damage in the city blocks is calculated. Finally, rescue teams can be sent to areas by considering the priorities of the decision maker.

\footnotetext{
${ }^{*}$ Corresponding Author
} 
As shown in Figure 1, the study area is the district 2 of Tehran with area of about 64 square kilometres and an estimated population of 640,000 persons in recent years.

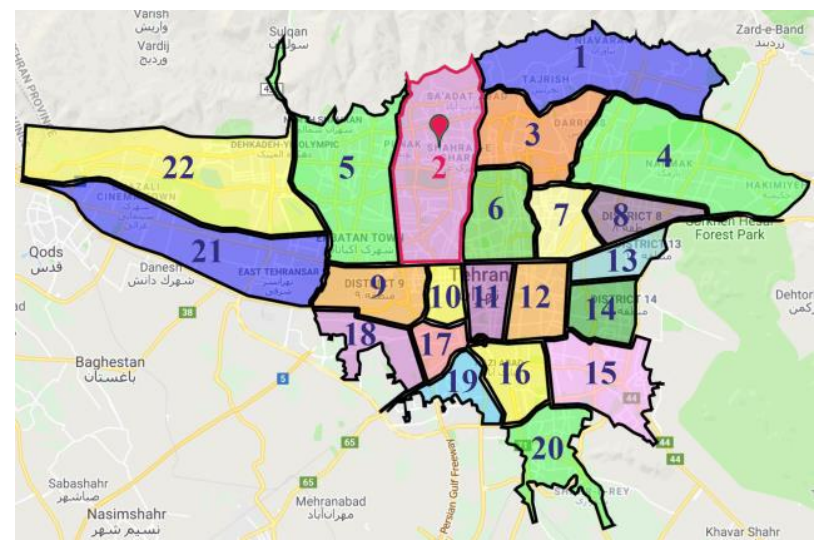

Figure 1. Districts of Tehran and location of district 2

To create a vulnerability map, characteristics of the buildings including the number of floors and the genus of buildings and geophysical specifications of the earth including the Peak Ground Acceleration (PGA) in terms of earthquake magnitude 6.5 in the study area as well as the locations of active faults in the area are used as the parameters affecting the amount of damage to the buildings by the earthquake. Initially, it is necessary to prepare the research data (criteria maps) in order to determine the rate of damage of urban features.

- Fault distance layer: The closer the building is to a fault, the more likely it is to be destroyed. As shown in the Figure 2, the farthest distance from the fault in the area is 3780 meters.

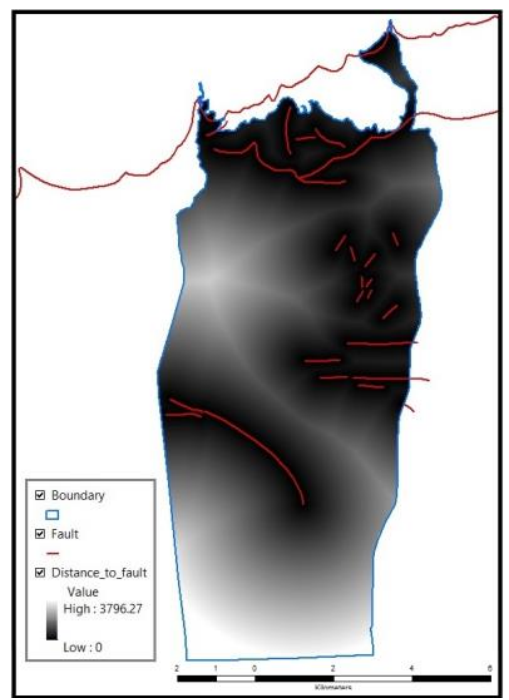

Figure 2. Distance to fault

- Peak ground acceleration layer: Peak Ground Acceleration (PGA) is equal to the maximum ground acceleration that occurs during earthquake shaking at a location. According to the data obtained from Iran Strong Motion (Road, Housing \& Urban Development Research Center) PGA varies from $0.21 \mathrm{~g}$ to $0.34 \mathrm{~g}$ in the study area (Figure 3 ). The higher the PGA value, the greater the probability of damage.

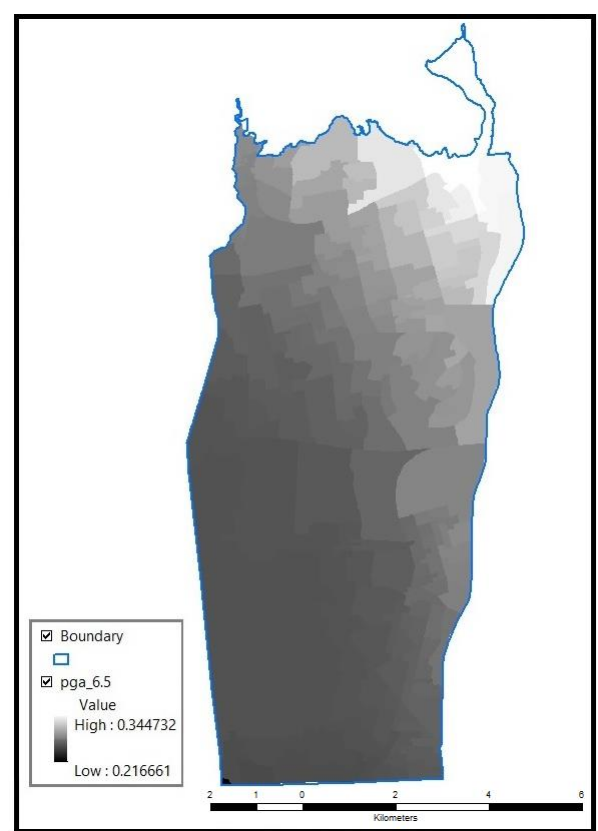

Figure 3. PGA map

- Number of floors in the building layer: This map shows the number of floors of the buildings in the study area .Figure 4 shows that its range is between 0 floors (the parcels without any building) to 36 floors. The taller the building, the more vulnerable it is.

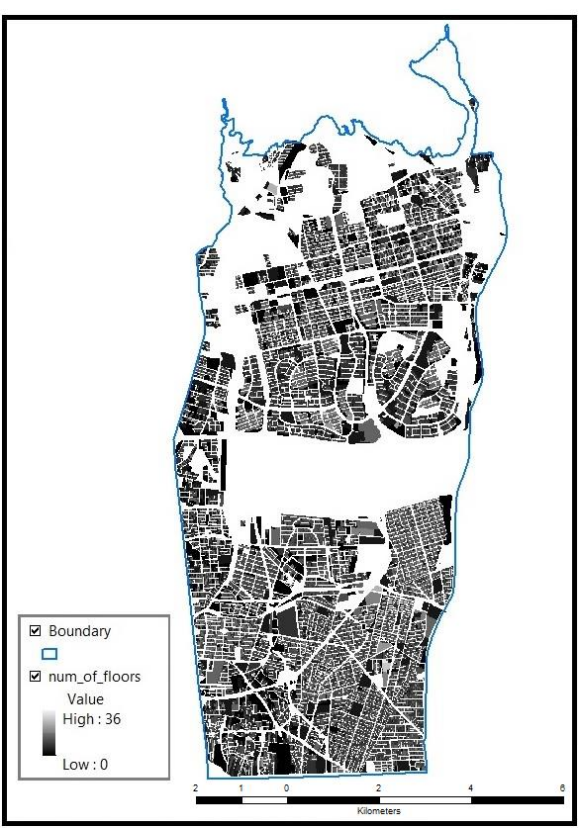

Figure 4. Number of floors

- Building structure type layer: The buildings in the study area classification into 4 classes of concrete, metal, masonry, adobe and wood (Figure 5). The amount of damage to each building depends on the strength of the building. 


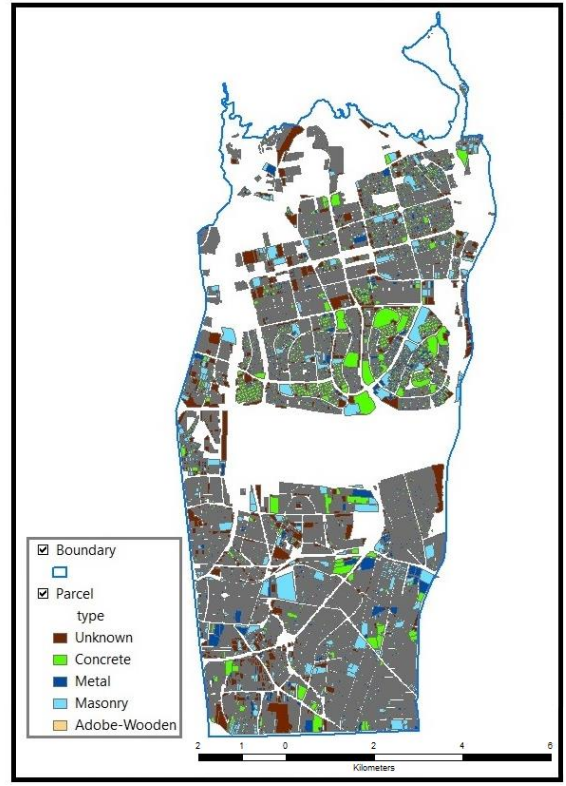

Figure 5. Structure types of the buildings

To use the fuzzy inference in MATLAB software, the input parameters and membership functions for each of them were defined:

- Three qualitative classes of "low", "medium" and "high" are used to design fault distance membership functions and also to determine the effect of this parameter on the seismic hazard of structures. As shown in Figure 6 trapezoidal continuous functions are used to design the membership functions of these three sets.

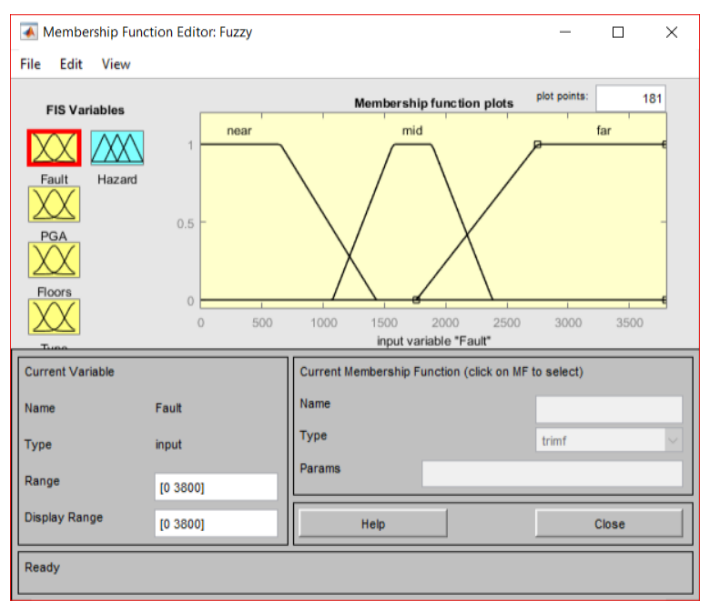

Figure 6. Fault distance membership functions

- Triangular membership function is used to define the peak ground acceleration or PGA membership functions. It is designed so that each function overlaps with its adjacent function. As shown in Figure 7 three accelerations of $0.21 \mathrm{~g}, 0.27 \mathrm{~g}$ and $0.34 \mathrm{~g}$ (at the top of the triangles) are defined in the low, medium and high range.

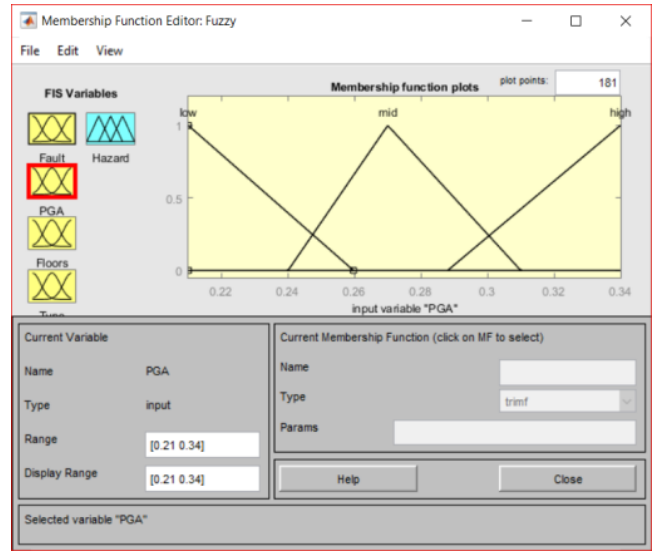

Figure 7. PGA membership functions

- The short, mid and tall trapezoid membership functions is used for number of floors of the buildings (Figure 8).

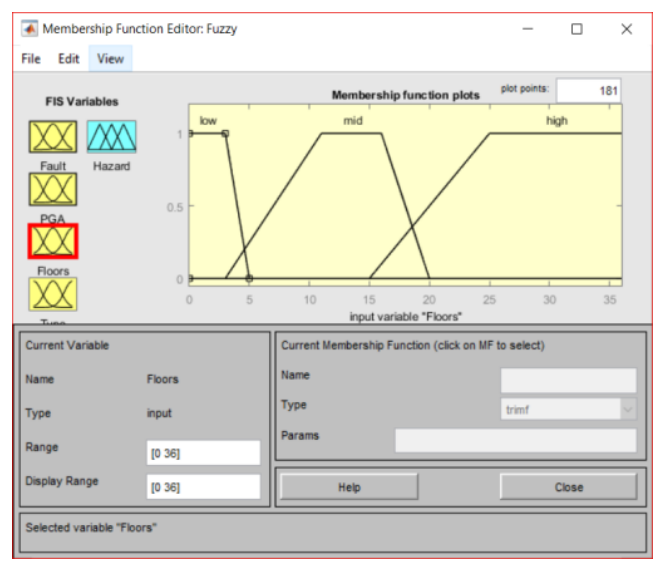

Figure 8. Number of floors' membership functions

- As illustrated in Figure 9 the trapezoidal membership function is also used for the buildings structure type, but the trapezoid is used as a rod for any of the types of metal, concrete, masonry, brick and wood around the defined number. Adobe and wood with the lowest earthquake resistance in the last class, followed by masonry, concrete and metal are moving towards greater resistance.

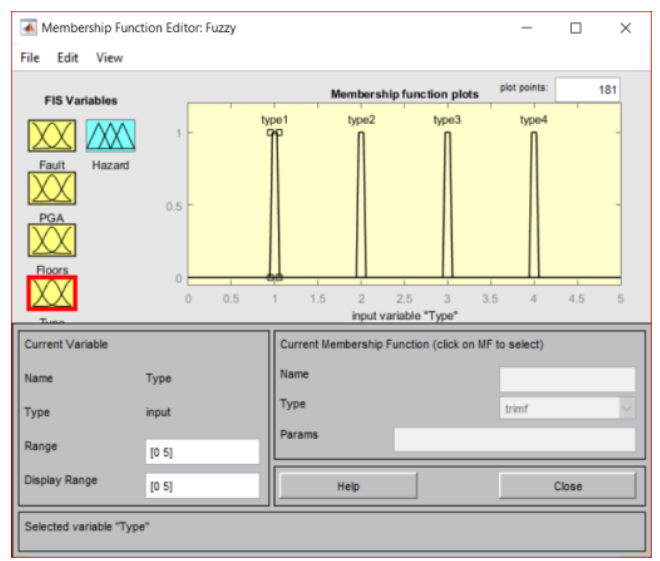

Figure 9. Membership functions of structure type 
Damage membership function is defined in Gaussian form include three classes of low, medium and high. As shown in Figure 10, the Gaussian functions are symmetrically used to design their membership functions. The reason for this type of design is to increase the design accuracy, because in most cases the output of the model is not an integer and can be a decimal number.

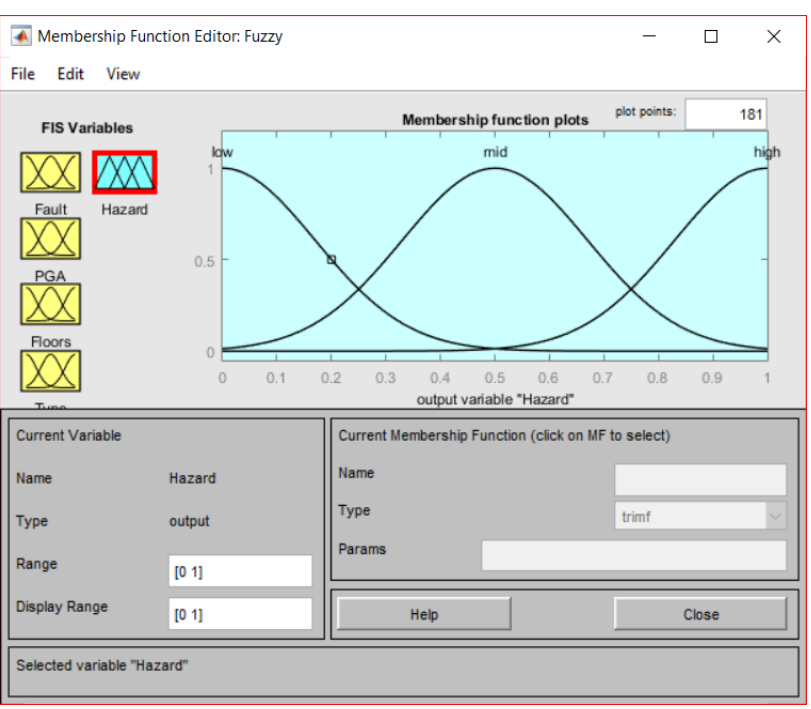

Figure 10. Damage membership function

To complete the fuzzy inference system, the set of if-then conditional rules must be written. By reviewing existing datasets, as well as scientific logical relationships, several conditional rules were written. These rules were optimized in terms of efficiency and accuracy. Some examples of written ifthen rules can be seen in Figure 11. The inference method follows Mamdani inference system (Allali et al., 2018).

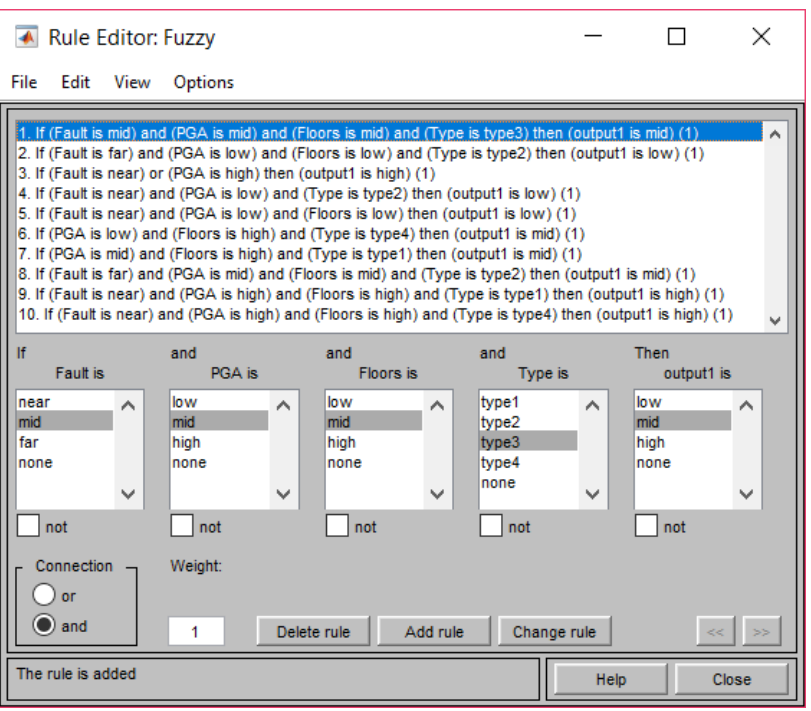

Figure 11. Employed fuzzy rules ranging from the highest vulnerable to the lowest vulnerable area (Figure 12).

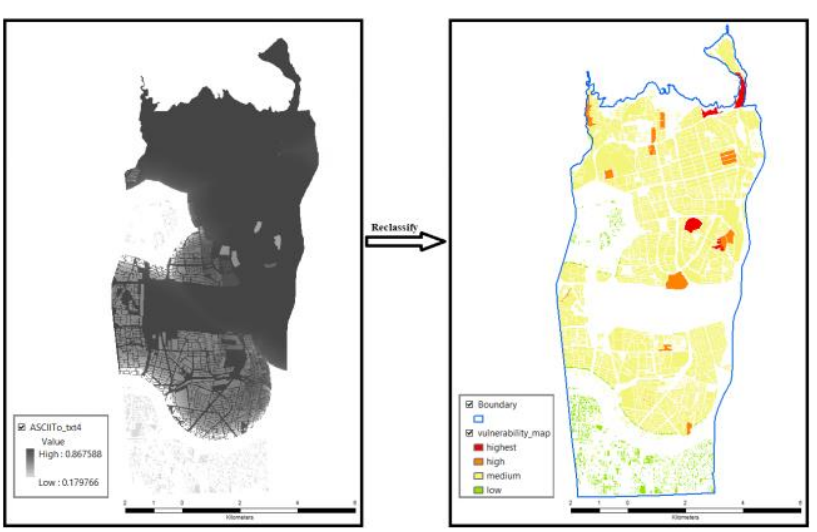

Figure 12. Vulnerability Map

The next step of this research is to determine the best locations in the study area for establishment emergency internet supplier instruments.

In order to establish internet communication in times of crisis, using radio wireless devices is an appropriate and cost-effective suggestion (Frenzel, 2018). Mikrotik Wireless Radio, with a powerful antenna, will provide seamless communication. Using this instrument hand-over is done automatically and without any interruption. The antenna is a passive device and the radio is an active device. Due to their output power, radios are capable of transmitting signals to a limited extent, but adding an antenna can significantly improve both signal reception and transmission power. In other words, antennas are a complementary device to radios.

During the research, Mikrotik DynaDish 5 is a cheap $5 \mathrm{GHz}$ device designed for outdoor use and equipped with a Gigabit Ethernet port to take full advantage of $802.11 \mathrm{ac}$ standard power. Theoretically, using a $5 \mathrm{GHz}$ device with $802.11 \mathrm{ac}$ standard provides the fastest data transfer rate, at speeds of about 800 Mbps (Shin and Bagchi, 2013).

This device is one of the most ideal instruments for point-topoint connections over distances up to 15 kilometers. DynaDish 5 's body and cover are made from durable plastic; therefore this radio is able to make lasting connections in harsh weather conditions and is a good choice for high frequency contamination due to the antenna cover (Chen et al., 2019). Wireless radios are able to communicate within a certain range, which is considered to be circular.

Due to the $15 \mathrm{~km}$ board of Mikrotik DynaDish 5, only two wireless radios can provide internet access in the study area in times of crisis. Therefore, the south-north extends of the area (as the largest dimension) divided into two equal parts and the geometric centre of each part was determined as the position of wireless radio (Figure 13).

The result of applying fuzzy rules on the fuzzy factor maps is a vulnerability map. Then the map was classified into four classes 


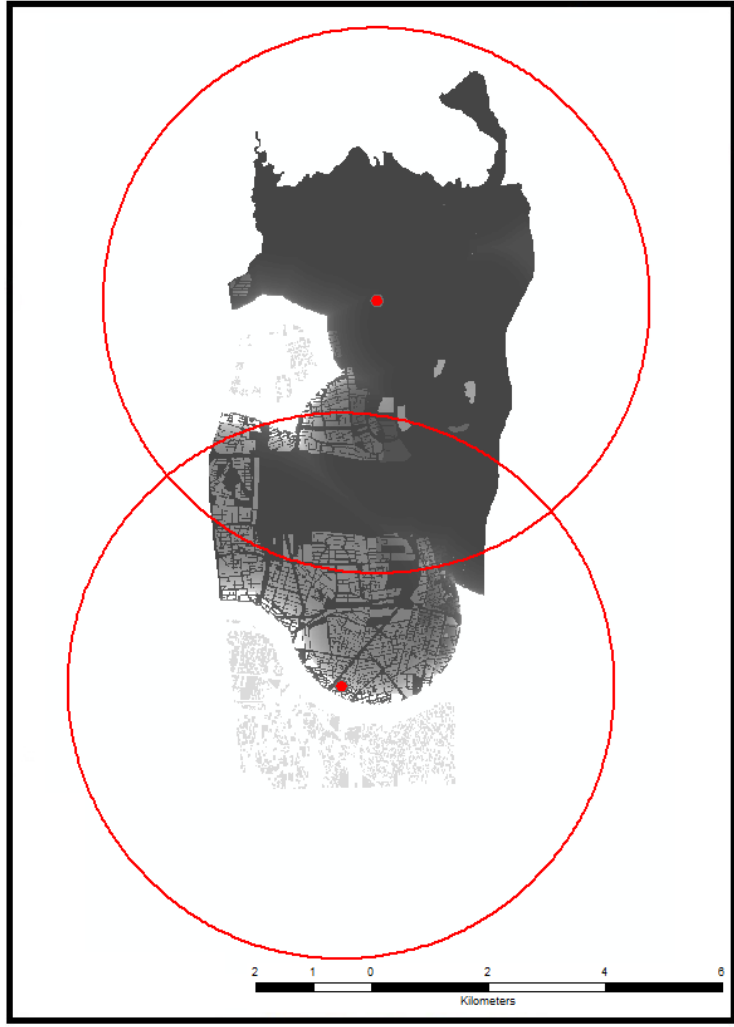

Figure 13. Range of Mikrotik DynaDish 5 in Region

In this research, the effort is to provide relief more quickly and minimize human and material damage. One of the things we need in times of severe crisis is to quickly record the location and report the location of an event. Now if the location, without the need for special training, is sent in high-precision numerical coordinates by the people in the crisis areas it will have an effective contribution to the accuracy and speed of decisionmaking. Using VGI through mobile phones helps identify the affected areas hidden in the created vulnerability map. This simulation can be used alongside other expert systems as an appropriate information and analytical tool for management decisions regarding earthquake damage reduction.

For this purpose, an application is written in the Android Studio environment that collects crisis reports with the help of people in disaster areas. Android is an operating system for mobile devices. It is mostly used for smartphones, like Google's own Google Pixel, as well as by other phone manufacturers like HTC and SAMSUNG. A modified Linux kernel is used as Android's kernel.

The app is designed to allow users to easily submit reports after connecting to the internet network, even if their phone cannot be locating. In the following, the details of this app will be explained.

As shown in Figure 14, to ensure the information received first, it is necessary to register name and mobile number.

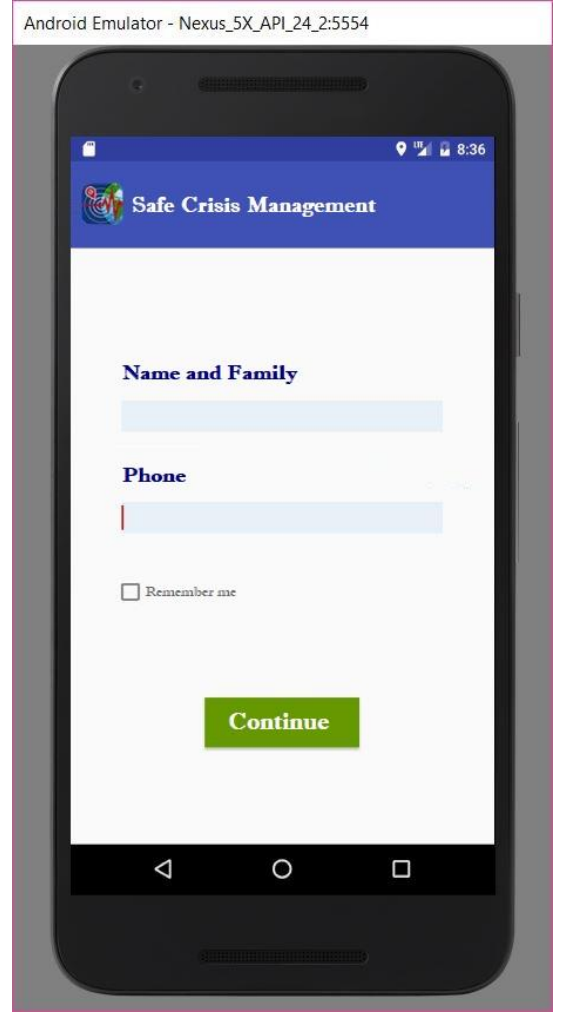

Figure 14. Registration page

Then, the location of the person is displayed on the map if it is in a location where it is possible to locate. As mentioned, due to the possibility of lack of location services in smartphones during the crisis, it's necessary that the app be designed in such a way that the person can save locations before the crisis. So if the locating is not available, the page of the saved places will appear to the user (Figure 15).

To save a location before the crisis, you need to select add to favorite button on map page (Figure 16). After the user has determined the point either on the map or through the saved points, the user must determine the type of damage. As shown in Figure 17, one has to choose an option so that the decision center can categorize the received reports and then handle them.

All user-defined specifications are stored in the user's mobile SQLite database, which is then sent to the decision center by touching the send button and confirming the reliability of the recorded report (Figure 18). Ultimately, the decision-making center collects voluntary information from people affected by the use of mobile smartphones, providing a comprehensive view of the extent of the damage in the area and can make better and faster decisions 


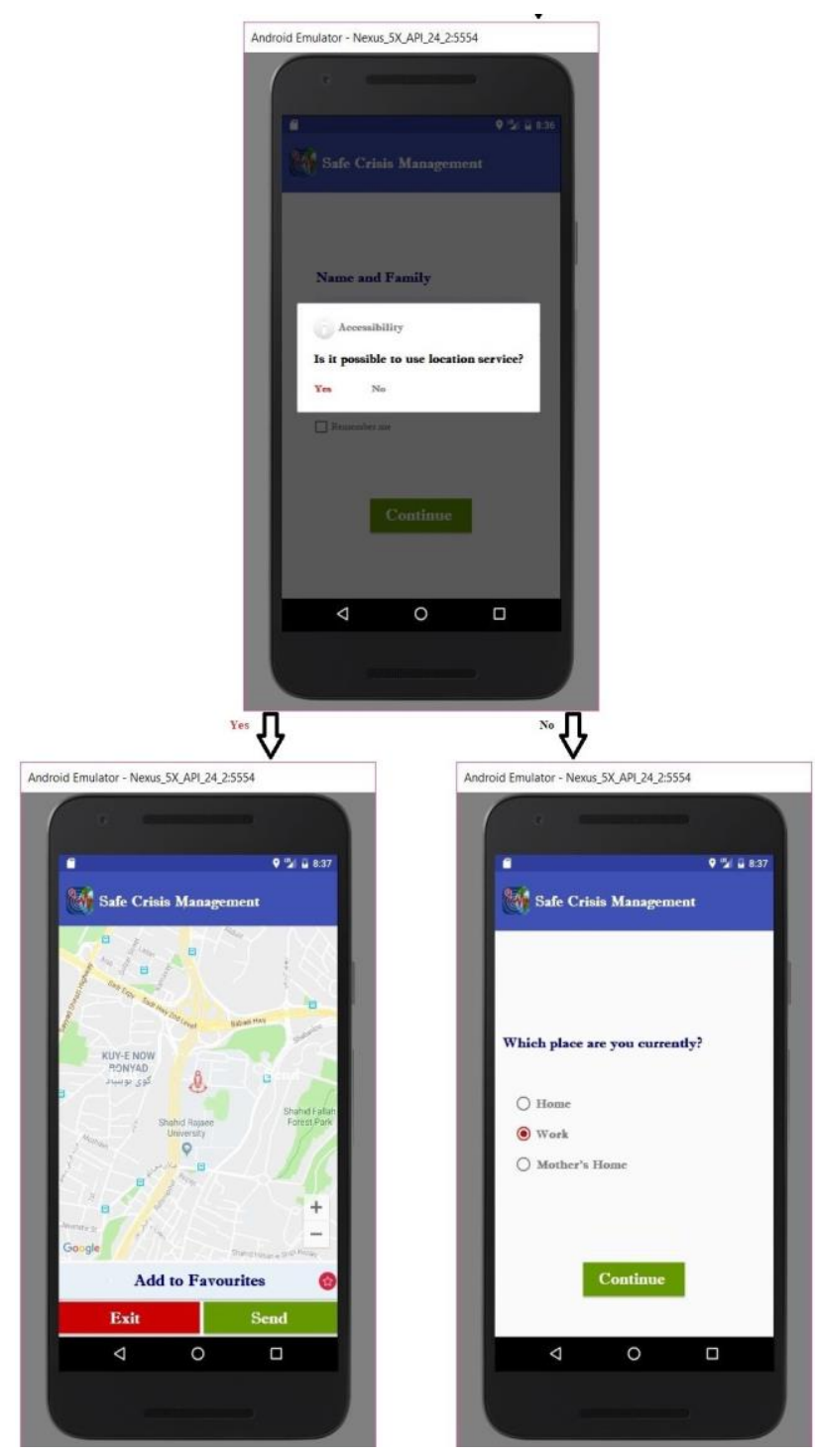

Figure 15. Location page (Using location service if possible or select saved location if not possible)

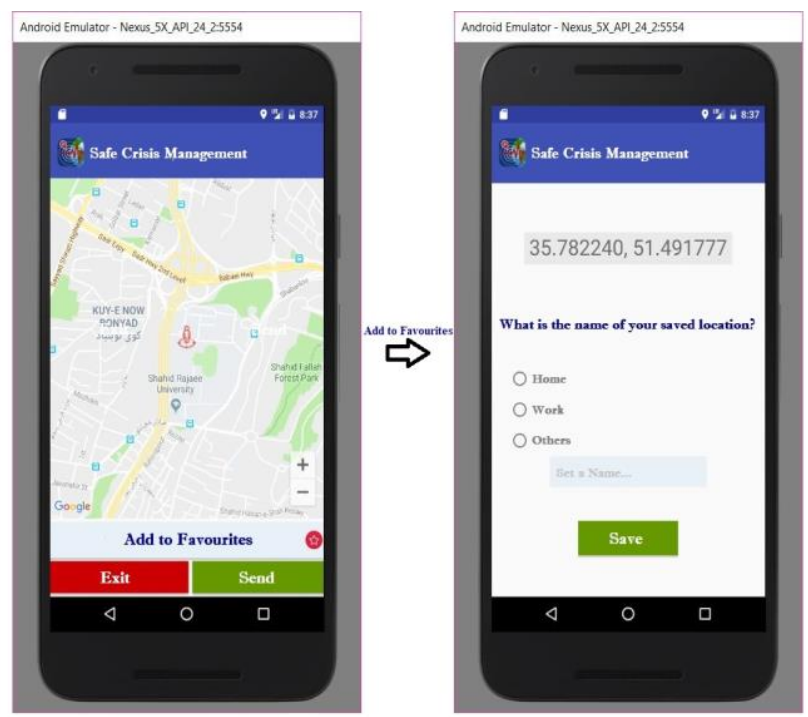

Figure 16. Save location

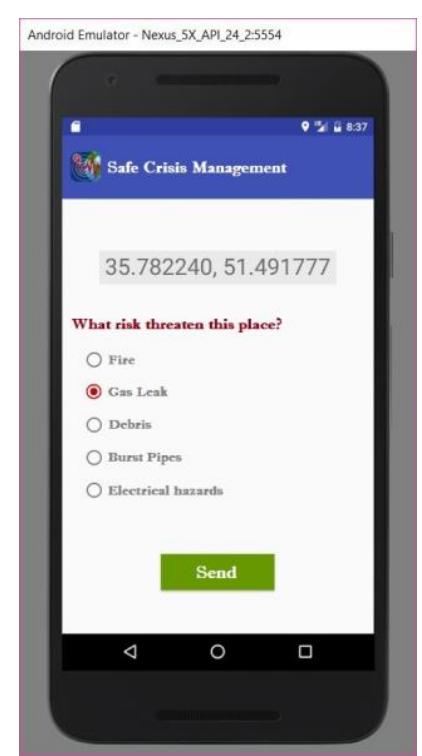

Figure 17. Determine the damage

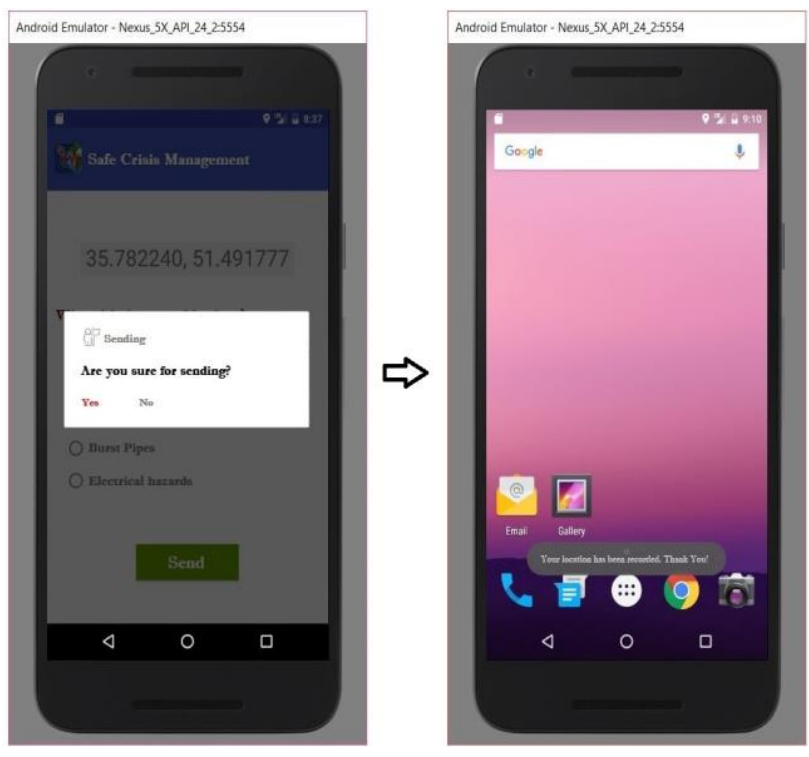

Figure 18. Verification of report

\section{RESULTS}

The main result of this study is the implementation of a simulation environment for post-earthquake relief allocation. This simulation is based on determining the extent of damage in an earthquake-prone city. In the simulation, it was attempted to approximate the data used in the present situation after the earthquake. For this purpose, fuzzy inference system was used among different methods to evaluate seismic vulnerability of the city. On the other hand, people's voluntary reports are used to gain a better understanding of the situation after the crisis and identify hidden areas. For this purpose, it was necessary to provide internet in the area and program a practical app for people. Based on this research also the following consequences were achieved:

- Based on the provided vulnerability map, urban planners and managers are able to design plans for crisis management or revise the previous ones. 
- The building and population vulnerability map and associated reports illustrate the various damage classes in the region, providing an opportunity to the managers to prioritize building reinforcement plans and prepare educational programs to prepare people for the time of disasters.

- $\quad$ This research offers an inexpensive method for people at the time of crisis to ask for help including their location even they are heavily damaged.

- Volunteer peoples' data collected with mobile GIS, helps us to observe the hidden dangers in the region and reduce the amount of damage by responding quickly.

Generally, this model can be suitable and efficient for use in hazard mitigation as well as estimation of required resource in the disaster management cycle.

\section{CONCLUSIONS}

In fact, the results clearly demonstrate the high vulnerability of the region and the fragility of its urban texture. The use of GIS in this work has allowed the exploitation of the data collected in every situation and the diagnosis of the most vulnerable zones. A spatial analysis has permitted to locate geographically the structures and rates of damages as well as their typology. Through this study, now we know the vulnerable regions in the study area. These results may also be useful in organization of the first aids, reinforcement of buildings and reconstruction's actions.

This paper showed that GIS-based analysis is a useful approach for earthquake disaster management - before, during and after the occurrence of an earthquake.

Providing people with a user friendly application for asking help or reporting the situations can play a critical role in the crisis management process. From a cost-benefit point of view, the method is a beneficial alternative for formal data collection methods as it not only cuts down on the time of data collection process but also fundamentally reduces the cost of preparing required equipment by only an easily available application. However, using peoples' smart phones as data collection tools in crisis management process such as earthquake disaster, basically requires high tech geo-informatics infrastructures such as comprehensive geodatabases at fine resolution, equipped with strong hardware and rapid software.

\section{REFERENCES}

Abdalla, R., 2016. Mobile GIS and location-based services (LBS). In Introduction to Geospatial Information and Communication Technology (GeoICT) (pp. 83-103). Springer, Cham.

Alavi Razavi, A., 2008. Bam Earthquake Report. Kerman Disaster Management Center.

Allali, S.A., Abed, M., Mebarki, A., 2018. Post-earthquake assessment of buildings damage using fuzzy logic. Engineering Structures 166, 117-127.

Chen, L., Abdellatif, S., Simo Tegueu, Armel F., Gayraud, T., 2019. Embedding and re-embedding of virtual links in software-defined multi-radio multi-channel multi-hop wireless networks. Computer Communications 145, 161-175.
Frenzel, L.E., 2018. Chapter 7 - Radio/Wireless: The Invisible Cables of Modern Electronics, in: Frenzel, L.E. (Ed.), Electronics Explained (Second Edition). Newnes, pp. 159-194.

Gao, S., Mai, G., 2018. Mobile GIS and Location-Based Services. Reference Module in Earth Systems and Environmental Sciences 2018, 384-397.

Geng, J., Song, W., Sun, S., 2016. A Study on Crowdsourcing Geospatial Data Mining Based on Spatial Statistics. In International Conference on Energy, Power and Electrical Engineering.

Goetz, M., Zipf, A., 2013. The Evolution of GeoCrowdsourcing: Bringing Volunteered Geographic Information to the Third Dimension. In: Sui D., Elwood S., Goodchild M. (eds) Crowdsourcing Geographic Knowledge. Springer, Dordrecht, pp. 139-159.

Goodchild, M.F., 2007a. Citizen as Voluntary Sensors: Spatial Data Infrastructure in the World of Web 2.0. International Journal of Spatial Data Infrastructures Research 2, 24-32.

Goodchild, M.F., 2007b. Citizens as sensors: the world of volunteered geography. GeoJournal 69, 211-221.

Hassanzadeh, R., Nedović- Budić, Z., Alavi Razavi, A., Norouzzadeh, M., Hodhodkian, H., 2013. Interactive approach for GIS-based earthquake scenario development and resource estimation (Karmania hazard model). Computers \& Geosciences 51, 324-338.

Hudson-Smith, A., Batty, M., Crooks, A., Milton, R.W., 2008. Mapping for the Masses: Accessing Web 2.0 through Crowdsourcing. Social Science Computer Review, 27, 524-538.

Lemmens, M., 2011. Mobile GIS and Location-Based Services. Nadim, F., Moghtaderi-Zadeh, M., Lindholm, C., Andresen, A., Remseth, S., Bolourchi, M.J., Mokhtari, M., Tvedt, E., 2004. The Bam Earthquake of 26 December 2003. Bulletin of Earthquake Engineering 2, 119-153.

Peterson, M., 2013. Crowdsourcing Geographic Knowledge. Daniel Sui, Sarah Elwood, and Michael Goodchild, eds. The AAG Review of Books 1, 125-126.

Shin, D.-H., Bagchi, S., 2013. An optimization framework for monitoring multi-channel multi-radio wireless mesh networks. Ad Hoc Networks 11, 926-943.

Zare, M., Kamran Zad, F., 2015. A Study on the Seismicity of Iran. Journal of Spatial Analysis Environmental Hazarts 1, 3958. 